J. DIFFERENTIAL GEOMETRY

83 (2009) 27-42

\title{
HAMILTONIAN STATIONARY SHRINKERS AND EXPANDERS FOR LAGRANGIAN MEAN CURVATURE FLOWS
}

\author{
YNG-ING LEE \& Mu-TAO WANG
}

\begin{abstract}
We construct examples of shrinkers and expanders for Lagrangian mean curvature flows. These examples are Hamiltonian stationary and asymptotic to the union of two Hamiltonian stationary cones found by Schoen and Wolfson in [SWO]. The SchoenWolfson cones $C_{p, q}$ are obstructions to the existence problems of special Lagrangians or Lagrangian minimal surfaces in the variational approach. It is known that these cone singularities cannot be resolved by any smooth oriented Lagrangian submanifolds. The shrinkers and expanders that we found can be glued together to yield solutions of the Brakke motion-a weak formulation of the mean curvature flow. For any coprime pair $(p, q)$ with $p>q>1$, we construct such a solution that resolves one single Schoen-Wolfson cone $C_{p, q}$. Note that $C_{p, q}$ is stable only if $p-q=1$. It thus provides an evidence to Schoen-Wolfson's conjecture that the $(2,1)$ cone is the only area-minimizing cone. Higher dimensional generalizations are also obtained.
\end{abstract}

\section{Introduction}

The existence of special Lagrangians in Calabi-Yau manifolds received much attention recently due to the critical role it plays in the T-duality formulation of Mirror symmetry of Strominger-Yau-Zaslow [SYZ]. Schoen and Wolfson took up the variational approach of constructing special Lagrangians by minimizing volumes in suitable Lagrangian classes. They discovered non-flat Lagrangian cones that are Hamiltonian stationary [SWO]. The existence of special Lagrangians can be established once these cone singularities are excluded. However, these singularities do occur in the Lagrangian minimizers in some K-3 surfaces, see [WO]. Another potential approach to the construction of special Lagrangians is the mean curvature flow- as the negative gradient flow of the volume functional. However, the long-time existence of

The first author is supported by Taiwan NSC grant 95-2115-M-002. The second author is supported by NSF grant DMS0605115 and a Sloan research fellowship.

Received 07/02/07. 
such flows can only be verified in some special cases, see for example [SM], [SWA], [WA1], and [WA2]. In this article, we construct special weak solutions of the Lagrangian mean curvature flows and show that the union of two related Schoen-Wolfson cones can be resolved by these flows.

Our ambient space is always the complex Euclidean space $\mathbb{C}^{n}$ with coordinates $z^{i}=x^{i}+\sqrt{-1} y^{i}$, the standard symplectic form $\omega=\sum_{i=1}^{n} d x^{i} \wedge$ $d y^{i}$, and the standard almost complex structure $J$ with $J\left(\frac{\partial}{\partial x^{i}}\right)=\frac{\partial}{\partial y^{i}}$. On a Lagrangian submanifold $\Sigma$, the mean curvature vector $H$ is given by

$$
H=J \nabla \beta
$$

where $\beta$ is the Lagrangian angle and $\nabla$ is the gradient on $\Sigma$. By the first variation formula, the mean curvature vector points to the direction where the volume is decreased most rapidly. In this case, $\beta$ can be defined by the relation that

$$
*_{\Sigma}\left(d z^{1} \wedge \cdots \wedge d z^{n}\right)=e^{i \beta}
$$

where $*_{\Sigma}$ is the Hodge ${ }^{*}$-star operator on $\Sigma$. We recall:

Definition 1.1. A Lagrangian submanifold $\Sigma$ is called Hamiltonian stationary if the Lagrangian angle is harmonic. i.e. $\Delta \beta=0$ where $\Delta$ is the Laplace operator on $\Sigma$. $\Sigma$ is a special Lagrangian if $\beta$ is a constant function.

A Hamiltonian stationary Lagrangian submanifold is a critical point of the volume functional among all Hamiltonian deformations and a special Lagrangian is a volume minimizer in its homology class.

As the special Lagrangians are volume minimizers, it is thus natural to use the mean curvature flow in the construction of special Lagrangians. Equation (1.1) implies that the mean curvature flow is a Lagrangian deformation, i.e. a Lagrangian submanifold remains Lagrangian along the mean curvature flow. In a geometric flow, the singularity often models on a soliton solution. In the case of mean curvature flows, one type of soliton solutions of particular interest are those moved by scaling in the Euclidean space. We recall:

Definition 1.2. A Lagrangian submanifold in the Euclidean space is called a self-similar solution if

$$
F^{\perp}=2 c H
$$

for some constant $c$, where $F^{\perp}$ is normal projection of the position vector $F$ in the Euclidean space and $H$ is the mean curvature vector. It is called a self-shrinker if $c<0$ and self-expander if $c>0$. 
It is not hard to see that if $F$ is a self-similar solution, then $F_{t}$ defined by $F_{t}=\sqrt{\frac{t}{c}} F$ is moved by the mean curvature flow. By Huisken's monotonicity formula $[\mathbf{H U}]$, any central blow up of a finite-time singularity of the mean curvature flow is a self-similar solution. In this article, we find Hamiltonian stationary self-shrinkers and self-expanders of the Lagrangian mean curvature flow that are asymptotic to the union of two Schoen-Wolfson cones. Altogether they form a Brakke flow (see $\S 3$ ) which is a weak formulation of the mean curvature flow proposed by Brakke in $[\mathbf{B R}]$. To be more precise, we prove:

Theorem 1.1. For each Schoen-Wolfson cone $C_{p q}$, there exists a corresponding Schoen-Wolfson cone $C_{p q}^{\prime}$ and a solution $V_{t},-\infty<t<\infty$ of the Brakke motion without mass loss so that $V_{t}, t<0$ is a smooth Hamiltonian stationary self-shrinker and $V_{t}, t>0$ is a smooth Hamiltonian stationary self-expander. Moreover, $V_{t}$ approach the union $C_{p q} \cup C_{p q}^{\prime}$ as $t \rightarrow 0$ from either direction.

Definition 1.3. We call such a solution $V_{t},-\infty<t<\infty$, a Hamiltonian stationary self-similar eternal Brakke motion.

Without loss of generality, we can assume that $p>q$. When $q>1$, we show that a single Schoen-Wolfson cone can be resolved by self-similar Brakke motion.

Theorem 1.2. For any Schoen-Wolfson cone $C_{p, q}$ with $p>q>1$, there exists a Hamiltonian stationary self-similar eternal Brakke motion $V_{t}$ such that $V_{t}$ approaches $C_{p, q}$ as $t \rightarrow 0$ from either direction.

Schoen and Wolfson show that $C_{p, q}$ is (Hamiltonian) stable only if $p-q=1$ and they conjecture that only the $C_{2,1}$ cone is area-minimizing (in oriented Lagrangian). The first author were informed by R. Schoen that this is the first time when $C_{2,1}$ can be distinguished from all other stable $C_{p, q}$ cones.

We remark that self-similar solutions of Lagrangian mean curvature flows were constructed by Anciaux $[\mathbf{A N}]$ using a different ansatz. His examples approach special Lagrangian cones while ours approach Hamiltonian stationary cones. Haskins [HA3] also observed these solutions are Hamiltonian stationary based on a Hamiltonian formulation similar to the one used by Harvey and Lawson $[\mathbf{H L}]$ in their construction of examples of special Lagrangians. Special Lagrangians and Hamiltonian stationary Lagrangians are constructed by many authors.

Our theorem is analogous to the Feldman-Ilmanen-Knopf $[\mathbf{F I K}]$ gluing construction for the Kähler-Ricci flows. Unlike the mean curvature flow, a notion of weak solutions of Ricci flow has not yet been established.

This article is organized as the follows. Our examples are presented in $\S 2$ and the formulation of Brakke motion is recalled in $\S 3$. In section 
$\S 4$ and $\S 5$, we prove theorem 1.1 and theorem 1.2, respectively. Higher dimensional examples are presented in $\S 6$.

Acknowledgments. Both authors thank Mark Haskins for enlightening conversations and comments on this subject. They would like to thank Dominic Joyce for referring them to the articles [JO1] and [JO2] and helpful remarks. The first author owes her gratitude to Tom Ilmanen for his inspiring discussions that lead to the finding of the corresponding cones and she thanks the hospitality of Ilmanen and Institute for Mathematical Research at ETH during her visit. The second author wishes to thank the support of the Taida Institute for Mathematical Sciences during the preparation of this article.

\section{Examples of self-similar solutions}

2.1. Schoen-Wolfson cones. Let $p$ and $q$ be two co-prime positive integers. The close and embedded curve

$$
\gamma_{p q}(\theta)=\left(\sqrt{\frac{q}{p+q}} e^{i p \theta}, i \sqrt{\frac{p}{p+q}} e^{-i q \theta}\right), \quad 0 \leq \theta<2 \pi
$$

is Legendrian and Hamiltonian stationary in $S^{3}$. The cone over $\gamma_{p q}(\theta)$ is Lagrangian and Hamiltonian stationary, and is denoted by $C_{p q}$. It is stable if and only if $|p-q|=1$. As such properties are invariant under $U(2)$, the cone over any $U(2)$ image of $\gamma_{p q}$ is again Lagrangian and Hamiltonian stationary. These are possible cone singularities for the Lagrangian minimizers studied in [SWO].

2.2. Self-shrinkers and self-expanders. We take the same ansatz as Schoen-Wolfson and consider the surfaces

$$
F(\mu, \theta)=\left(z_{1}(\mu) e^{i p \theta}, z_{2}(\mu) e^{-i q \theta}\right),
$$

where $0 \leq \theta<2 \pi, \mu \in \mathbb{R}$, and $z_{1}(\mu)$ and $z_{2}(\mu)$ are curves in the complex plane. A direct computation shows that a sufficient condition for $F(\mu, \theta)$ to be Lagrangian is that $p\left|z_{1}(\mu)\right|^{2}-q\left|z_{2}(\mu)\right|^{2}$ is a constant. One can further investigate the condition for $F(\mu, \theta)$ to be Hamiltonian stationary, and the condition for $F(\mu, \theta)$ to be self-similar. We will not explore the general situation here. Instead we show directly in the following that the surface

$$
F(\mu, \theta)=\left(\cosh \mu \sqrt{q} e^{i p \theta}, i \sinh \mu \sqrt{p} e^{-i q \theta}\right),
$$

where $0 \leq \theta<2 \pi$ and $\mu \in \mathbb{R}$, is Hamiltonian stationary and self-similar. We compute

$$
\frac{\partial F}{\partial \mu}=\left(\sinh \mu \sqrt{q} e^{i p \theta}, i \cosh \mu \sqrt{p} e^{-i q \theta}\right),
$$


and

$$
\frac{\partial F}{\partial \theta}=\sqrt{p q}\left(i \cosh \mu \sqrt{p} e^{i p \theta}, \sinh \mu \sqrt{q} e^{-i q \theta}\right) .
$$

It is easy to check that $\left\langle J \frac{\partial F}{\partial \mu}, \frac{\partial F}{\partial \theta}\right\rangle=0$, and thus the surface is Lagrangian. The components of the induced metric on the surface are

$$
\begin{gathered}
g_{11}=\left|\frac{\partial F}{\partial \mu}\right|^{2}=p \cosh ^{2} \mu+q \sinh ^{2} \mu, \\
g_{22}=\left|\frac{\partial F}{\partial \theta}\right|^{2}=p q\left(p \cosh ^{2} \mu+q \sinh ^{2} \mu\right),
\end{gathered}
$$

and $g_{12}=0$. Therefore the area form is given by

$$
\sqrt{p q}\left(p \cosh ^{2} \mu+q \sinh ^{2} \mu\right) d \mu d \theta
$$

A simple calculation shows that the Lagrangian angle $\beta=(p-q) \theta$. Thus $\Delta_{g} \beta=0$, it follows that the surface is Hamiltonian stationary. On a Lagrangian submanifold, we have the mean curvature vector $H=$ $J \nabla \beta$. Since $\beta$ depends only on $\theta$,

$$
H=\frac{1}{g_{22}} J \frac{\partial \beta}{\partial \theta} \frac{\partial F}{\partial \theta}=\frac{p-q}{g_{22}} J \frac{\partial F}{\partial \theta} .
$$

To calculate $F^{\perp}$ we note that the normal bundle of the surface is spanned by $J \frac{\partial F}{\partial \mu}$ and $J \frac{\partial F}{\partial \theta}$. We compute

$$
\left\langle F, J \frac{\partial F}{\partial \mu}\right\rangle=R e(-i q \cosh \mu \sinh \mu-i p \sinh \mu \cosh \mu)=0
$$

and

$$
\left\langle F, J \frac{\partial F}{\partial \theta}\right\rangle=\sqrt{p q} R e\left(-\cosh ^{2} \mu \sqrt{p q}+\sinh ^{2} \mu \sqrt{p q}\right)=-p q .
$$

Hence

$$
F^{\perp}=\frac{-p q}{g_{22}} J \frac{\partial F}{\partial \theta}=-\frac{p q}{p-q} H
$$

and $F$ is a self-similar solution. We summarize the calculations in this section in the following proposition.

Proposition 2.1. If $p>q$ are two co-prime positive integers, then

$$
S(\mu, \theta)=\left(\cosh \mu \sqrt{q} e^{i p \theta}, i \sinh \mu \sqrt{p} e^{-i q \theta}\right),
$$

where $0 \leq \theta<2 \pi$ and $\mu \in \mathbb{R}$, is a Hamiltonian stationary shrinker and

$$
E(\mu, \theta)=\left(\sinh \mu \sqrt{q} e^{i p \theta}, i \cosh \mu \sqrt{p} e^{-i q \theta}\right),
$$

where $0 \leq \theta<2 \pi$ and $\mu \in \mathbb{R}$, is a Hamiltonian stationary expander. $S$ satisfies $F^{\perp}=-2 c H$ while $E$ satisfies $F^{\perp}=2 c H$, where $c=\frac{p q}{2(p-q)}$. 
We notice that $E$ can be obtained by switching $p$ and $q$ in the expression for $S$, taking bar, and multiplying by $\left[\begin{array}{ll}0 & i \\ i & 0\end{array}\right]$. As $\mu \rightarrow+\infty$, both $S$ and $E$ approach the Schoen-Wolfson cone $C_{p q}$ over the curve $\gamma_{p q}$.

2.3. Asymptotics of the flow. By the remark in the introduction, we have $\sqrt{\frac{-t}{c}} S$ for $t<0$ is a smooth solution of the mean curvature flow, so is $\sqrt{\frac{t}{c}} E$ for $t>0$.

Proposition 2.2. If $p>q$ are two co-prime positive integers, then

$$
S_{t}(\mu, \theta)=\sqrt{\frac{-t}{c}}\left(\cosh \mu \sqrt{q} e^{i p \theta}, i \sinh \mu \sqrt{p} e^{-i q \theta}\right),
$$

for $t<0$ is a smooth solution of the mean curvature flow and so is

for $t>0$.

$$
E_{t}(\mu, \theta)=\sqrt{\frac{t}{c}}\left(\sinh \mu \sqrt{q} e^{i p \theta}, i \cosh \mu \sqrt{p} e^{-i q \theta}\right),
$$

Denote by $h\left(S_{t}\right)$ the mean curvature vector of $S_{t}$ and $d\left\|S_{t}\right\|$ the area element of $S_{t}$, then

$$
\begin{gathered}
\left|S_{t}\right|^{2}=\left(\frac{-t}{c}\right)\left(q \cosh ^{2} \mu+p \sinh ^{2} \mu\right), \\
\left|h\left(S_{t}\right)\right|^{2}=\left(\frac{c}{-t}\right) \frac{(p-q)^{2}}{p q} \frac{1}{p \cosh ^{2} \mu+q \sinh ^{2} \mu}, \\
d\left\|S_{t}\right\|=\left(\frac{-t}{c}\right) \sqrt{p q}\left(p \cosh ^{2} \mu+q \sinh ^{2} \mu\right) d \mu d \theta .
\end{gathered}
$$

For positive co-prime integers $p$ and $q$ with $p>q$, we define

$$
\begin{aligned}
& C_{++}(y, \theta)=\left(y \sqrt{q} e^{i p \theta}, i y \sqrt{p} e^{-i q \theta}\right), \\
& C_{+-}(y, \theta)=\left(y \sqrt{q} e^{i p \theta},-i y \sqrt{p} e^{-i q \theta}\right), \\
& C_{-+}(y, \theta)=\left(-y \sqrt{q} e^{i p \theta}, i y \sqrt{p} e^{-i q \theta}\right), \quad \text { and } \\
& C_{--}(y, \theta)=\left(-y \sqrt{q} e^{i p \theta},-i y \sqrt{p} e^{-i q \theta}\right),
\end{aligned}
$$

for $y \geq 0$ and $0 \leq \theta<2 \pi$. Here $C_{++}=C_{p q}$.

Note that $S_{t}$, as $t \rightarrow 0^{-}$, approaches $C_{++} \cup C_{+-}$while $E_{t}$, as $t \rightarrow 0^{+}$, approaches $C_{++} \cup C_{-+}$. The asymptotic cones of $S_{t}$ and $E_{t}$ do not match unless $p$ and $q$ are both odd. In other cases, we can modify $S_{t}$ and $E_{t}$ so their asymptotic cones agree at $t=0$. This allows us to construct a Brakke flow $F_{t}$ that is a Hamiltonian stationary self-shrinker for $t<0$, 
a Hamiltonian stationary self-expander for $t>0$, and a pair of cones at $t=0$ in all cases.

\section{Brakke motion}

A family of varifolds $V_{t}$ is said to form a solution of the Brakke motion $[\mathbf{B R}]$ if

$$
\bar{D}\left\|V_{t}\right\|(\phi) \leq \delta\left(V_{t}, \phi\right)\left(h\left(V_{t}\right)\right)
$$

for each $\phi \in C_{0}^{1}\left(\mathbb{R}^{n}\right)$ with $\phi \geq 0$, where $\bar{D}\left\|V_{t}\right\|(\phi)$ is the upper derivative defined by $\varlimsup_{t_{1} \rightarrow t} \frac{\left\|V_{t_{1}}\right\|(\phi)-\left\|V_{t}\right\|(\phi)}{t_{1}-t}$ and $h\left(V_{t}\right)$ is the generalized mean curvature vector of $V_{t}$. In the setting of this paper,

$$
\delta\left(V_{t}, \phi\right)\left(h\left(V_{t}\right)\right)=-\int \phi\left|h\left(V_{t}\right)\right|^{2} d\left\|V_{t}\right\|+\int D \phi \cdot h\left(V_{t}\right) d\left\|V_{t}\right\| .
$$

In our case, the singularity happens at the $t=0$ slice. We formulate the following proposition as a criterion to check the solutions of Brakke motion in this case.

Proposition 3.1. Suppose the varifold $V_{t}, a<t<b$ forms a smooth mean curvature flow in $\mathbb{R}^{n}$ except at $t=c \in(a, b)$ and $\left\|V_{t}\right\|$ converges in Radon measure to $\left\|V_{c}\right\|$ as $t \rightarrow c$. If $\lim _{t \rightarrow c^{-}} \frac{d}{d t}\left\|V_{t}\right\|(\phi)$ and $\lim _{t \rightarrow c^{+}} \frac{d}{d t}\left\|V_{t}\right\|(\phi)$ are both either finite or $-\infty$ and

$$
\lim _{t \rightarrow c^{ \pm}} \frac{d}{d t}\left\|V_{t}\right\|(\phi) \leq \delta\left(V_{0}, \phi\right)\left(h\left(V_{0}\right)\right)
$$

for any $\phi \in C_{0}^{1}\left(\mathbb{R}^{n}\right)$ then $V_{t}$ forms a solution of the Brakke motion.

Proof. Since $\left\|V_{t}\right\|(\phi)$ is continuous on the interval $(a, c]$ and differentiable on $(a, c)$. By the mean value theorem, we have

$$
\lim _{t \rightarrow c^{-}} \frac{\left\|V_{t}\right\|(\phi)-\left\|V_{c}\right\|(\phi)}{t-c}=\lim _{t \rightarrow c^{-}} \frac{d}{d t}\left\|V_{t}\right\|(\phi) .
$$

The case for $t \rightarrow c^{+}$can be treated similarly. Therefore the assumption (3.2) implies that (3.1) holds.

q.e.d.

\section{Proof of Theorem 1.1}

The proof of Theorem 1.1 is divided into three cases according to the parities of $p$ and $q$. In each case, we show that (3.2) holds with equality.

Before going into the details of the proof, we first make some observations on the asymptotic cones. Consider shrinkers of the form

$$
\left\{\sqrt{\frac{-t}{c}}\left(x_{1} \sqrt{q} e^{i p \theta}, i x_{2} \sqrt{p} e^{-i q \theta}\right): x_{1}^{2}-x_{2}^{2}=1, t<0\right\}
$$


and expanders

$$
\left\{\sqrt{\frac{t}{c}}\left(x_{1} \sqrt{q} e^{i p \theta}, i x_{2} \sqrt{p} e^{-i q \theta}\right): x_{1}^{2}-x_{2}^{2}=-1, t>0\right\} .
$$

As $t \rightarrow 0$ both of them converge to $C_{++} \cup C_{+-} \cup C_{-+} \cup C_{--}$. By shifting $\theta$ to $\theta+\pi$, it is easy to see that

(i) When both $p$ and $q$ are odd, $C_{++}=C_{--}$and $C_{+-}=C_{-+}$.

(ii) When $p$ is odd and $q$ is even, $C_{++}=C_{-+}$and $C_{+-}=C_{--}$.

(iii) When $p$ is even and $q$ is odd, $C_{++}=C_{+-}$and $C_{-+}=C_{--}$.

That is, the shrinkers and expanders converge to the double of two cones. In the following, we manage to arrange $S_{t}$ and $E_{t}$ so that they converge to a single copy of the two cones. More precisely, when $p$ and $q$ are both odd, the asymptotic cones are $C_{++} \cup C_{+-}$. When $p$ is odd and $q$ is even, the asymptotic cones are $C_{++} \cup C_{+-}$. When $p$ is even and $q$ is odd, the asymptotic cones are $C_{++} \cup C_{-+}$.

4.1. Case 1: both $p$ and $q$ are odd. We start with $t<0$. By change of variable $y=\sqrt{\frac{-t}{c}} \sinh \mu$, it is not hard to see $S_{t}$ as $t \rightarrow 0^{-}$converges to the varifold $S_{0}$ defined by

$$
S_{0}(y, \theta)=\left(|y| \sqrt{q} e^{i p \theta}, i y \sqrt{p} e^{-i q \theta}\right), \quad y \in \mathbb{R}, 0 \leq \theta<2 \pi .
$$

The norm square of $S_{0}$ is given by

$$
\left|S_{0}\right|^{2}=y^{2}(p+q) .
$$

The norm square of generalized mean curvature vector and the area element of $S_{0}$ are given by:

$$
\left|h\left(S_{0}\right)\right|^{2}=\frac{(p-q)^{2}}{p q(p+q)} \frac{1}{y^{2}}
$$

and

$$
d\left\|S_{0}\right\|=|y| \sqrt{p q}(p+q) d y d \theta .
$$

Since for a smooth mean curvature flow, we have

$$
\frac{d}{d t}\left\|S_{t}\right\|(\phi)=\delta\left(S_{t}, \phi\right)\left(h\left(S_{t}\right)\right)=-\int \phi\left|h\left(S_{t}\right)\right|^{2} d\left\|S_{t}\right\|+\int D \phi \cdot h\left(S_{t}\right) d\left\|S_{t}\right\| .
$$

To apply Proposition 3.1, it suffices to show

$$
\begin{aligned}
& \lim _{t \rightarrow 0^{-}}-\int \phi\left|h\left(S_{t}\right)\right|^{2} d\left\|S_{t}\right\|+\int D \phi \cdot h\left(S_{t}\right) d\left\|S_{t}\right\| \\
& =-\int \phi\left|h\left(S_{0}\right)\right|^{2} d\left\|S_{0}\right\|+\int D \phi \cdot h\left(S_{0}\right) d\left\|S_{0}\right\|
\end{aligned}
$$


and the limit is either finite or $-\infty$. From (2.3) and (2.4), we obtain

$$
\left|h\left(S_{t}\right)\right|^{2} d\left\|S_{t}\right\|=\frac{(p-q)^{2}}{\sqrt{p q}} d \mu d \theta
$$

and

$$
\left|h\left(S_{t}\right)\right| d\left\|S_{t}\right\|=(p-q) \sqrt{\left(\frac{-t}{c}\right)\left(p \cosh ^{2} \mu+q \sinh ^{2} \mu\right)} d \mu d \theta .
$$

We first show that $\int D \phi \cdot h\left(S_{t}\right) d\left\|S_{t}\right\|$ is always finite. By (4.6), this integral is bounded above by

$$
\begin{aligned}
& \int|D \phi|\left|h\left(S_{t}\right)\right| d\left\|S_{t}\right\| \\
& =(p-q) \int\left|D \phi\left(S_{t}(\mu, \theta)\right)\right| \sqrt{\left(\frac{-t}{c}\right)\left(p \cosh ^{2} \mu+q \sinh ^{2} \mu\right)} \mu d \theta .
\end{aligned}
$$

Suppose $\phi$ vanishes outside $B(0 ; R)$ and recall the expression $(2.2)$ for $\left|S_{t}\right|$, we see the integral is supported in the domain

$$
\left(\frac{-t}{c}\right)\left(q \cosh ^{2} \mu+p \sinh ^{2} \mu\right) \leq R^{2}, \quad 0 \leq \theta<2 \pi .
$$

Thus (4.7) is bounded above by

$$
C_{1} \int_{\left(\frac{-t}{c}\right)\left(q \cosh ^{2} \mu+p \sinh ^{2} \mu\right) \leq R^{2}} \sqrt{\left(\frac{-t}{c}\right)\left(p \cosh ^{2} \mu+q \sinh ^{2} \mu\right)} d \mu
$$

for some constant $C_{1}>0$ depending on the upper bound of $|D \phi|$.

Consider the change of variable $y=\sqrt{\frac{-t}{c}} \sinh \mu$, we have

$$
d y=\sqrt{\frac{-t}{c}} \cosh \mu d \mu \quad \text { and } \quad \sqrt{\frac{-t}{c}} \cosh \mu=y \frac{\cosh \mu}{\sinh \mu} .
$$

Hence (4.8) becomes

$$
C_{1} \int_{|y| \leq \sqrt{\frac{R^{2}+\left(\frac{t}{c}\right) q}{p+q}}} \sqrt{p+q \tanh ^{2} \mu} d y
$$

which is finite as $\tanh ^{2} \mu \leq 1$.

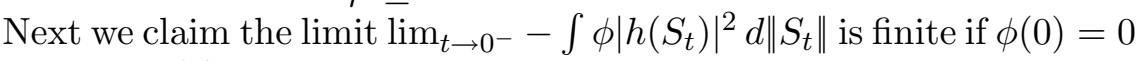
and $-\infty$ if $\phi(0) \neq 0$.

By (4.5),

$$
\int \phi\left|h\left(S_{t}\right)\right|^{2} d\left\|S_{t}\right\|=\int \phi\left(S_{t}(\mu, \theta)\right) \frac{(p-q)^{2}}{\sqrt{p q}} d \mu d \theta .
$$


When $\phi(0)=0$, we may assume $\phi$ is supported in $B(0 ; R)$ and $\phi\left(S_{t}(\mu, \theta)\right) \leq C_{2}\left|S_{t}(\mu, \theta)\right|$ for some $C_{2}>0$, therefore

$$
\begin{aligned}
& \int \phi\left(S_{t}(\mu, \theta)\right) d \mu d \theta \\
\leq & C_{3} \int_{\left(\frac{-t}{c}\right)\left(q \cosh ^{2} \mu+p \sinh ^{2} \mu\right) \leq R^{2}} \sqrt{\left(\frac{-t}{c}\right)\left(q \cosh ^{2} \mu+p \sinh ^{2} \mu\right)} d \mu
\end{aligned}
$$

for some $C_{3}>0$.

This is similar to (4.7) and can be shown to be finite by the change of variable $y=\sqrt{\frac{-t}{c}} \sinh \mu$.

On the other hand, when $\phi(0)>0$, we may assume $\phi(0) \geq C_{4}>0$ on $B(0 ; \epsilon)$, thus

$$
\begin{aligned}
\int \phi\left(S_{t}(\mu, \theta)\right) d \mu d \theta & \geq 2 \pi C_{4} \int_{\left(\frac{-t}{c}\right)\left(q \cosh ^{2} \mu+p \sinh ^{2} \mu\right) \leq \epsilon^{2}} d \mu \\
& =2 \pi C_{4} \int_{|y| \leq \sqrt{\frac{\epsilon^{2}+\frac{t}{c} q}{p+q}}} \frac{1}{\sqrt{y^{2}+\left(\frac{-t}{c}\right)}} d y
\end{aligned}
$$

which tends to $\infty$ as $t \rightarrow 0^{-}$by observing $\frac{1}{\sqrt{y^{2}+\left(\frac{-t}{c}\right)}} \geq \frac{1}{|y|+\sqrt{\frac{-t}{c}}}$.

Equations (4.1), (4.2), and (4.3) imply that $\int \phi\left|h\left(S_{0}\right)\right|^{2} d\left\|S_{0}\right\|$ is finite if $\phi(0)=0$ and $-\infty$ if $\phi(0)>0$. Now (4.4) follows from the change of variable $y=\sqrt{\frac{-t}{c}} \sinh \mu$, the fact that $h\left(S_{t}\right) \rightarrow h\left(S_{0}\right)$, and the dominant convergence theorem.

For $t>0$, we consider $E_{t}$ with

$$
\begin{gathered}
\left|E_{t}\right|^{2}=\left(\frac{t}{c}\right)\left(p \cosh ^{2} \mu+q \sinh ^{2} \mu\right), \\
\left|h\left(E_{t}\right)\right|^{2}=\left(\frac{c}{t}\right) \frac{(p-q)^{2}}{p q} \frac{1}{q \cosh ^{2} \mu+p \sinh ^{2} \mu}, \\
d\left\|E_{t}\right\|=\left(\frac{t}{c}\right) \sqrt{p q}\left(q \cosh ^{2} \mu+p \sinh ^{2} \mu\right) d \mu d \theta .
\end{gathered}
$$

As $t \rightarrow 0^{+}, E_{t}$ converges to the varifold $E_{0}$ defined by

$$
E_{0}(y, \theta)=\left(y \sqrt{q} e^{i p \theta}, i|y| \sqrt{p} e^{-i q \theta}\right), \quad y \in \mathbb{R}, 0 \leq \theta<2 \pi .
$$

$E_{0}$ coincides with $S_{0}$ by the change of variable

$$
E_{0}(y, \theta)=S_{0}(y, \theta+\arg y) .
$$

when $p$ and $q$ are both odd (note that $\arg y=0$ or $\pi$ ). 
The identity

$$
\begin{aligned}
& \lim _{t \rightarrow 0^{+}}-\int \phi\left|h\left(E_{t}\right)\right|^{2} d\left\|E_{t}\right\|+\int D \phi \cdot h\left(E_{t}\right) d\left\|E_{t}\right\| \\
& =-\int \phi\left|h\left(E_{0}\right)\right|^{2} d\left\|E_{0}\right\|+\int D \phi \cdot h\left(E_{0}\right) d\left\|E_{0}\right\|
\end{aligned}
$$

can be checked similarly.

4.2. Case 2: $p$ odd and $q$ even. In this case, for $t<0, V_{t}$ is defined to be $S_{t}$ as before. Thus by change of variable $y=\sqrt{\frac{-t}{c}} \sinh \mu, S_{t}$ as $t \rightarrow 0^{-}$converges to the varifold $S_{0}$ defined by

$$
S_{0}(y, \theta)=\left(|y| \sqrt{q} e^{i p \theta}, i y \sqrt{p} e^{-i q \theta}\right), \quad y \in \mathbb{R}, 0 \leq \theta<2 \pi .
$$

Moreover, the identity (4.4) holds.

For $t>0$, we define $V_{t}$ to be $e^{i \arg \mu} E_{t}\left(\mu, \theta+\frac{\arg \mu}{q}\right)$ (note that $\arg \mu=$ 0 or $\pi$ ). By change of variable $y=\sqrt{\frac{t}{c}} \sinh \mu$, it is not hard to see $V_{t}$ as $t \rightarrow 0^{+}$converges to the varifold $V_{0}$ defined by

$V_{0}(y, \theta)= \begin{cases}\left(y \sqrt{q} e^{i p \theta}, i y \sqrt{p} e^{-i q \theta}\right), & y \geq 0,0 \leq \theta<2 \pi \\ -\left(y \sqrt{q} e^{i p\left(\theta+\frac{\pi}{q}\right)}, i|y| \sqrt{p} e^{-i q\left(\theta+\frac{\pi}{q}\right)}\right), & y<0,0 \leq \theta<2 \pi\end{cases}$

$V_{0}$ coincides with $S_{0}$ by the change of variable

$$
V_{0}(y, \theta)=S_{0}\left(y, \theta+\frac{\arg y}{q}\right) .
$$

The angle shift of $V_{t}$ for $\mu<0$ is to make the parametrization continuous at $\mu=0$. Although the tangent plane from $\mu \rightarrow 0^{+}$and $\mu \rightarrow 0^{-}$ do not agree. The induced volume form and mean curvature vector from both sides are the same. Hence $V_{t}$ can still be considered as a selfexpander for $t>0$. In fact, the image of $V_{t}$ for $t>0$ can be regarded as two complete non-oriented smooth surfaces intersecting at one circle. We claim that when $V_{t}$ is considered as a Radon measure, its generalized mean curvature vector $h\left(V_{t}\right)$ is the same as the usual mean curvature vector. That is, there is no contribution from the singular set $\{\mu=0\}$. To compute the generalized mean curvature vector, we choose a family of ambient diffeomorphism $\psi_{s}$ with $\psi_{0}=i d$ and $\left.\frac{d \psi_{s}}{d s}\right|_{s=0}=W$ and derive the first variation formula $\left.\frac{d\left\|\left(\psi_{s}\right)_{*}\left(V_{t}\right)\right\|}{d s}\right|_{s=0}$.

We can divide the image into $\mu<0$ and $\mu>0$ with boundary curve $\left\{\sqrt{\frac{t}{c}}\left(0, i \sqrt{p} e^{-i q \theta}\right): 0 \leq \theta<2 \pi\right\}$, and calculate separately. To prove the claim, the essential part is to compute the contribution from the boundary. Note that the unit normal of the boundary from the $\mu>0$ side 
is $\left(e^{i p \theta}, 0\right)$, while it is $\left(-e^{i p\left(\theta+\frac{\pi}{q}\right)}, 0\right)$ from the $\mu<0$ side. We observe that each of $\theta, \theta+\frac{2 \pi}{q}, \cdots, \theta+\frac{2 \pi(q-1)}{q}$ determines the same boundary point for $0 \leq \theta<\frac{2 \pi}{q}$. Thus the contribution of the boundary to the first variation from the $\mu>0$ side is

$$
\begin{aligned}
& \int_{0}^{2 \pi} W \cdot\left(e^{i p \theta}, 0\right) \sqrt{\frac{t p}{c}} d \theta \\
& =\sqrt{\frac{t p}{c}} \int_{0}^{\frac{2 \pi}{q}} W \cdot\left(e^{i p \theta}, 0\right)\left(1+e^{i \frac{2 \pi p}{q}}+\cdots+e^{i \frac{2 \pi p(q-1)}{q}}\right) d \theta=0 .
\end{aligned}
$$

The last equality follows from the fact that

$$
1+e^{i \frac{2 \pi p}{q}}+\cdots+e^{i \frac{2 \pi p(q-1)}{q}}=0
$$

for $p, q$ being two co-prime integers and $q>1$. Since $q$ is a positive even number, this is certainly the case. The contribution of the boundary from the $\mu<0$ side is also zero for the same reason. Thus the usual mean curvature vector agrees with the generalized mean curvature vector for $V_{t}$. We have

$$
\left|h\left(V_{t}\right)\right|^{2}=\frac{c}{t} \frac{(p-q)^{2}}{p q}\left(p \cosh ^{2} \mu+q \sinh ^{2} \mu\right)
$$

which is bounded for any fixed $t>0$. Thus by the dominate convergence theorem, we still have

$$
\frac{d}{d t}\left\|V_{t}\right\|(\phi)=-\int \phi\left|h\left(V_{t}\right)\right|^{2} d\left\|V_{t}\right\|+\int D \phi \cdot h\left(V_{t}\right) d\left\|V_{t}\right\|
$$

for $t>0$.

Hence to apply Proposition 3.1, it suffices to show

$$
\begin{aligned}
& \lim _{t \rightarrow 0^{+}}-\int \phi\left|h\left(V_{t}\right)\right|^{2} d\left\|V_{t}\right\|+\int D \phi \cdot h\left(V_{t}\right) d\left\|V_{t}\right\| \\
& =-\int \phi\left|h\left(V_{0}\right)\right|^{2} d\left\|V_{0}\right\|+\int D \phi \cdot h\left(V_{0}\right) d\left\|V_{0}\right\| .
\end{aligned}
$$

This identity can be checked similarly using the following equations:

$$
\begin{gathered}
\left|V_{t}\right|^{2}=\left(\frac{t}{c}\right)\left(p \cosh ^{2} \mu+q \sinh ^{2} \mu\right), \\
\left|h\left(V_{t}\right)\right|^{2}=\left(\frac{c}{t}\right) \frac{(p-q)^{2}}{p q} \frac{1}{q \cosh ^{2} \mu+p \sinh ^{2} \mu}, \\
d\left\|V_{t}\right\|=\left(\frac{t}{c}\right) \sqrt{p q}\left(q \cosh ^{2} \mu+p \sinh ^{2} \mu\right) d \mu d \theta .
\end{gathered}
$$


4.3. Case 3: $p$ even and $q$ odd. In this case, for $t>0, V_{t}$ is defined to be $E_{t}$ as in case 1 . Thus by change of variable $y=\sqrt{\frac{t}{c}} \sinh \mu, E_{t}$ as $t \rightarrow 0^{+}$converges to the varifold $E_{0}$ defined by

$$
E_{0}(y, \theta)=\left(y \sqrt{q} e^{i p \theta}, i|y| \sqrt{p} e^{-i q \theta}\right), \quad y \in \mathbb{R}, 0 \leq \theta<2 \pi .
$$

Moreover, the identity (4.13) holds.

For $t<0$, we define $V_{t}$ to be $e^{i \arg \mu} S_{t}\left(\mu, \theta+\frac{\arg \mu}{p}\right)$ (note that $\arg \mu=$

0 or $\pi$ ). By change of variable $y=\sqrt{\frac{-t}{c}} \sinh \mu$, it is not hard to see $V_{t}$ as $t \rightarrow 0^{-}$converges to the varifold $V_{0}$ defined by

$V_{0}(y, \theta)= \begin{cases}\left(y \sqrt{q} e^{i p \theta}, i y \sqrt{p} e^{-i q \theta}\right), & y \geq 0,0 \leq \theta<2 \pi \\ -\left(|y| \sqrt{q} e^{i p\left(\theta+\frac{\pi}{p}\right)}, i y \sqrt{p} e^{-i q\left(\theta+\frac{\pi}{p}\right)}\right), & y<0,0 \leq \theta<2 \pi\end{cases}$

$V_{0}$ coincides with $E_{0}$ by the change of variable

$$
V_{0}(y, \theta)=E_{0}\left(y, \theta+\frac{\arg y}{p}\right) .
$$

By similar discussions as in case 2, it can be shown that for $t<0, V_{t}$ is still a self-shrinker and satisfies (4.15).

Moreover, for $t<0$, we have $V_{t}$ with

$$
\begin{gathered}
\left|V_{t}\right|^{2}=\left(\frac{-t}{c}\right)\left(q \cosh ^{2} \mu+p \sinh ^{2} \mu\right), \\
\left|h\left(V_{t}\right)\right|^{2}=\left(\frac{c}{-t}\right) \frac{(p-q)^{2}}{p q} \frac{1}{p \cosh ^{2} \mu+q \sinh ^{2} \mu}, \\
d\left\|V_{t}\right\|=\left(\frac{-t}{c}\right) \sqrt{p q}\left(p \cosh ^{2} \mu+q \sinh ^{2} \mu\right) d \mu d \theta .
\end{gathered}
$$

The identity

$$
\begin{aligned}
& \lim _{t \rightarrow 0^{-}}-\int \phi\left|h\left(V_{t}\right)\right|^{2} d\left\|V_{t}\right\|+\int D \phi \cdot h\left(V_{t}\right) d\left\|V_{t}\right\| \\
& =-\int \phi\left|h\left(V_{0}\right)\right|^{2} d\left\|V_{0}\right\|+\int D \phi \cdot h\left(V_{0}\right) d\left\|V_{0}\right\|
\end{aligned}
$$

can be checked similarly.

\section{Proof of Theorem 1.2}

It is important to determine which cone constructed in $[\mathbf{S W O}]$ is area minimizing among Lagrangian competitors. Only these cones can occur as blow-up profiles for the singularities in the Lagrangian minimizers. Schoen and Wolfson show that a $(p, q)$ cone is (Hamiltonian) stable if 
and only if $p-q=1$ and conjectured that only $(2,1)$ cone (assuming $p>q$ ) is area minimizing. From the proof in section 4.2 , we can in fact show that this is the case infinitesimally. In the following, we prove Theorem 1.2.

Proof. Suppose $p>q>1$ and $\mu \geq 0,0 \leq \theta<2 \pi$. Define $V_{t}=$ $S_{t}(\mu, \theta)$ for $t<0, V_{t}=E_{t}(\mu, \theta)$ and $V_{0}=C_{p q}$. Both $S_{t}$ and $E_{t}$ converge to $C_{p q}$ as $t \rightarrow 0$. When $t \neq 0$, the image of $\mu=0$ is the boundary of $V_{t}$. Because both $p$ and $q$ are greater than one, there is no boundary contribution on the generalized mean curvature vector. We take the case $t>0$ as an example. The boundary curve is $\left\{\sqrt{\frac{t}{c}}\left(0, i \sqrt{p} e^{-i q \theta}\right): 0 \leq \theta<2 \pi\right\}$ and the unit normal vector is $\left(e^{i p \theta}, 0\right)$.

We observe that each of $\theta, \theta+\frac{2 \pi}{q}, \cdots, \theta+\frac{2 \pi(q-1)}{q}$ determines the same boundary point for $0 \leq \theta<\frac{2 \pi}{q}$. Thus the contribution from the boundary to the first variation is

$$
\begin{aligned}
& \int_{0}^{2 \pi} W \cdot\left(e^{i p \theta}, 0\right) \sqrt{\frac{t p}{c}} d \theta \\
& =\sqrt{\frac{t p}{c}} \int_{0}^{\frac{2 \pi}{q}} W \cdot\left(e^{i p \theta}, 0\right)\left(1+e^{i \frac{2 \pi p}{q}}+\cdots+e^{i \frac{2 \pi p(q-1)}{q}}\right) d \theta=0
\end{aligned}
$$

if $q$ is an integer greater than one. Because $p>1$, there is no contribution from the boundary to the first variation when $t<0$ either. The same arguments as in last section show that $V_{t}$ forms a solution of the Brakke motion.

q.e.d.

As a generalization of the mean curvature flow, the Brakke motion decreases area. Theorem 1.2 thus suggests that the cone $C_{p, q}$ may not be area minimizing when $q>1$. Wolfson's counterexample [WO] shows that one of the $(p, q)$ cone must be area minimizing. This leaves the $(2,1)$ cone as the only candidate for area minimizers. However, we remark this observation does not resolve the conjecture because, in classical sense, one needs to find Lagrangian competitors with the same boundary.

\section{Higher dimensional examples}

In the two-dimensional case, after multiplying by the matrix $\left[\begin{array}{cc}1 & 0 \\ 0 & -i\end{array}\right]$ $\in U(2)$, our example can be rewritten in the form

$$
\left\{\left(x_{1} e^{i p \theta}, x_{2} e^{-i q \theta}\right) \mid p x_{1}^{2}-q x_{2}^{2}=p q,\left(x_{1}, x_{2}\right) \in \mathbb{R}^{2}, 0 \leq \theta<2 \pi\right\} \subset \mathbb{C}^{2}
$$


Now consider the following generalization to higher dimensions: for any $n$ nonzero real numbers $\lambda_{1}, \cdots, \lambda_{n}$, consider the submanifold $\Sigma$ of $\mathbb{C}^{n}$ defined by

$$
\left\{\left(x_{1} e^{i \lambda_{1} \theta}, \cdots, x_{n} e^{i \lambda_{n} \theta}\right) \mid \sum_{i=1}^{n} \lambda_{i} x_{i}^{2}=C,\left(x_{1}, \cdots, x_{n}\right) \in \mathbb{R}^{n}\right\}
$$

for some constant $C$.

It is not hard to check that $\Sigma$ is Lagrangian in $\mathbb{C}^{n}$ with Lagrangian angle given by $\beta=\left(\sum_{i=1}^{n} \lambda_{i}\right) \theta+c$ for some constant $c$. Therefore $\Sigma$ is Hamiltonian stationary and is special if $\sum_{i=1}^{n} \lambda_{i}=0$. Such special Lagrangians were studied by Haskins in [HA1] [HA2] (for $n=3$ ) and Joyce in [JO1] (for general dimensions). We were also informed by Professor Joyce that the Hamiltonian stationary ones may also be obtained by applying his method of "perpendicular symmetries" in [JO2].

If $\sum_{i=1}^{n} \lambda_{i} \neq 0$, the position vector $F$ satisfies

$$
F^{\perp}=\frac{-C}{\sum_{i=1}^{n} \lambda_{i}} H
$$

That is, the submanifold $\Sigma$ is a Hamiltonian stationary self-similar solution of the mean curvature flow. Similar procedures as in this paper can be applied to show that when $\lambda_{i}$ are all integers,

$$
\Sigma_{t}=\left\{\left(x_{1} e^{i \lambda_{1} \theta}, \cdots, x_{n} e^{i \lambda_{n} \theta}\right) \mid \sum_{i=1}^{n} \lambda_{i} x_{i}^{2}=(-2 t) \sum_{i=1}^{n} \lambda_{i}\right\}
$$

with $\left(x_{1}, \cdots, x_{n}\right) \in \mathbb{R}^{n}$ and $0 \leq \theta<2 \pi$ form a Brakke flow without mass loss. We shall discuss further properties of these higher dimensional examples in a forthcoming paper $[\mathbf{L W}]$.

\section{References}

[AN] H. Anciaux, Construction of Lagrangian self-similar solutions to the mean curvature flow in $\mathbb{C}^{n}$. Geom. Dedicata 120 (2006), 37-48, MR 2252892, Zbl 1098.35074.

[BR] K.A. Brakke, The motion of a surface by its mean curvature. Mathematical Notes, Princeton University Press, 1978, MR 0485012, Zbl 0386.53047.

[FIK] M. Feldman, T. Ilmanen \& D. Knopf, Rotationally symmetric shrinking and expanding gradient Kähler-Ricci solitons. J. Differential Geom. 65 (2003), no. 2, 169-209, MR 2058261, Zbl 1069.53036.

[HL] R. Harvey \& H.B. Lawson, Calibrated geometries. Acta Math. 148 (1982), 48156, MR 0666108, Zbl 0584.53021.

[HA1] M. Haskins, Special Lagrangian cones. Amer. J. Math. 126 (2004), no. 4, 845-871, MR 2075484, Zbl 1074.53067.

[HA2] M. Haskins, The geometric complexity of special Lagrangian $T^{2}$-cones. Invent. Math. 157 (2004), no. 1, 11-70, MR 2135184, Zbl 1064.53032. 
[HA3] M. Haskins, personal communications.

[HU] G. Huisken, Asymptotic behavior for singularities of the mean curvature flow. J. Differential Geom. 31 (1990), no. 1, 285-299, MR 1030675, Zbl 0694.53005.

[JO1] D. Joyce, Constructing special Lagrangian $m$-folds in $\mathbb{C}^{m}$ by evolving quadrics. Math. Ann. 320 (2001), no. 4, 757-797, MR 1857138, Zbl 1085.53503.

[JO2] D. Joyce, Special Lagrangian $m$-folds in $\mathbb{C}^{m}$ with symmetries. Duke Math. J. 115 (2002), no. 1, 1-51, MR 1932324, Zbl 1023.53033.

[LW] Y.I. Lee \& M.T. Wang, Hamiltonian stationary cones and self-similar solutions in higher dimension. To appear in Trans. Amer. Math. Soc., also available at arXiv:0802.0359.

[SWO] R. Schoen \& J.G. Wolfson, Minimizing area among Lagrangian surfaces: the mapping problem. J. Differential Geom. 58 (2001), no. 1, 1-86, MR 1895348, Zbl 1052.53056 .

[SM] K. Smoczyk, Angle theorems for the Lagrangian mean curvature flow. Math. Z. 240 (2002), no. 4, 849-883, MR 1922733, Zbl 1020.53045.

[SWA] K. Smoczyk \& M.-T. Wang, Mean curvature flows of Lagrangians submanifolds with convex potentials. J. Differential Geom. 62 (2002), no. 2, 243-257, MR 1988504, Zbl 1070.53042.

[SYZ] A. Strominger, S.-T. Yau \& E. Zaslow, Mirror symmetry is T-duality. Nuclear Phys. B 479 (1996), no. 1-2, 243-259, MR 1429831, Zbl 0998.81091.

[WA1] M.-T. Wang, Deforming area preserving diffeomorphism of surfaces by mean curvature flow. Math. Res. Lett. 8 (2001), no. 5-6, 651-662, MR 1879809, Zbl 1081.53056 .

[WA2] M.-T. Wang, Long-time existence and convergence of graphic mean curvature flow in arbitrary codimension. Invent. Math. 148 (2002), no. 3, 525-543, MR 1908059, Zbl 1039.53072.

[WO] J.G. Wolfson, Lagrangian homology classes without regular minimizers, J. Differential Geom. 71 (2005), 307-313, MR 2197143, Zbl 1097.53052.

Department of MATHEMATICS AND Taida Institute of Mathematical Sciences

NATIONAL TAIWAN UnivERSiTy

TAIPEI, TAIWAN

E-mail address: yilee@math.ntu.edu.tw

DEPARTMENT OF MATHEMATICS Columbia University NEW YoRK, NY 10027

E-mail address: mtwang@math.columbia.edu 